\title{
Sprache unter Beobachtung
}

\section{Anne-Françoise Allaz}

Prof. Dr. med., Mitglied der Redaktion

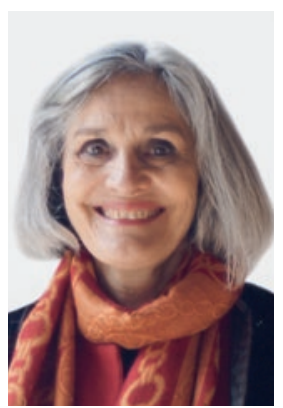

Seit geraumer Zeit werden die Debatten um eine «politisch korrekte» Sprache in europäischen Medien immer hitziger geführt. Hintergrund dieser Debatten ist das Aufkommen der Woke Culture und ihrer Verfechterinnen und Verfechter, die in Fragen rassistischer, genderbezogener und religiöser Diskriminierung sensibel («wach») sind.

Zur Verteidigung der Rechte von «Minderheiten» gehört auch, dass diskriminierende Aspekte unserer Sprache auf den Prüfstand kommen. So wird vielerorts bereits darauf geachtet, gendergerecht zu formulieren sowie anstelle von Bezeichnungen wie taub besser gehörlos oder anstelle von behindertengerecht besser barrierefrei zu verwenden. All diese Entwicklungen sind Ausdruck eines Wunsches nach Entstigmatisierung und Anerkennung.

Die von der Woke-Bewegung inspirierten Übertreibungen im Bereich der Identität sind rasch ins Zentrum politischer Diskussionen geraten. "Politisch korrekt» ist zu einem Vorwurf, ja sogar zu einer Beleidigung geworden. Zeigt sich darin Moral oder bereits Zensur? Es wird behauptet, durch eine übertriebene Aufwertung von Minderheiten und den Kommunitarismus bestünde die Gefahr, dass gemeinsame, auf die Aufklärung zurückgehende Normen aufgelöst würden. Glücklicherweise sehen viele Menschen den Schutz von Minderheiten als durchaus vereinbar mit Meinungsfreiheit und gemeinsamen Werten. Dennoch lässt sich feststellen, dass Sprache mittlerweile unter enormer Beobachtung steht. Worte sind schliesslich mächtig! Nach Michel Foucault sind die Art, wie wir über die Welt sprechen, und die Art, wie wir sie erleben, untrennbar miteinander verbunden.

Drei Beispiele unter zahlreichen anderen zeigen auf, wie stark der medizinische Bereich davon betroffen ist.

- Ein amerikanischer Spitalneurologe beklagt sich in JAMA [1] über die erhaltene Empfehlung, in den Krankenakten jede Beschreibung von Rasse, ethnischer Zugehörigkeit oder Herkunftsland der Patientinnen und Patienten zu vermeiden. Ich denke mir, dass hier die soziale Anamnese einer "politisch korrekten» Norm geopfert wird, wodurch medizinisch nützliche Informationen bedauernswerterweise auf der Strecke bleiben. Doch ein aktueller Lancet-Artikel weist mich streng zurecht [2]: Die Angabe von Rasse/ethnischer Zugehörigkeit in Konsultationsnotizen müsse überdacht werden, da es sich um strukturellen Rassismus im Gesundheitssystem handele. Ich bin perplex: «Patientin, 25 Jahre, Flüchtling, äthiopischer Herkunft» - das soll diskriminierend, abwertend sein? Das erschliesst sich mir nun wirklich nicht. Die Angabe der Herkunft der betreffenden Patientin gibt Aufschluss über ihre medizinisch-psychosoziale Situation und ermöglicht beispielsweise, eine entsprechend lange Beratungszeit einzuplanen.

- Ein weiteres Beispiel: Unter der Trump-Regierung wurde das Center for Disease Control (CDC) Ende 2017 aufgefordert, die Verwendung von sieben Begriffen in offiziellen Texten und Budgetanträgen zu vermeiden, darunter «vulnerabel», «Diversität», «Transgender», «Fötus» sowie «evidenz- und wissenschaftsbasiert» [3]. Die stark ideologische Natur einer solchen Weisung liegt auf der Hand, ebenso wie ihre Folgen für die Ausrichtung des öffentlichen Gesundheitswesens und der Forschung. Dieser Versuch, Wörter verschwinden zu lassen, erinnert an den von George Orwell in seinem Buch 1984 beschriebenen Neusprech, bei dem Wörter, die nicht dem Einheitsdenken entsprechen, ausgemerzt werden: «Begreifst $d u$ denn nicht, dass Neusprech nur ein Ziel hat, nämlich den Gedankenspielraum einzuengen?»

- Letztendlich komme ich zu dem Schluss, dass der technokratische Neusprech mit seinem Kontrollwunsch an Einfluss gewinnen könnte, wenn wir nicht wachsam bleiben. Er weist uns bereits die Rolle von Dienstleistungserbringern für Kunden zu, was der Komplexität der Beziehung zwischen Ärztinnen/Ärzten und Patientinnen/Patienten ganz und gar nicht gerecht wird.

Für mich stellt die Medizin weiterhin einen Raum dar, in dem (noch) alles - oder sagen wir fast alles - gesagt werden darf, aber falls nötig geheim bleibt. Es ist klar, dass wir in der Praxis ein Gleichgewicht zwischen Redefreiheit und gegenseitigem Respekt wahren und versuchen müssen, Diversität und Vulnerabilität in ihren unterschiedlichen Erscheinungsformen mit grösstmöglicher Toleranz und Wohlwollen zu begegnen. Dies ist Teil des Hippokratischen Eides.

Unsere Meinungsfreiheit und somit auch die Freiheit unserer Gedanken sind vor diesem Hintergrund ein ebenso kostbares Gut wie unsere Persönlichkeit und Kreativität. Lassen wir uns ihrer um keinen Preis berauben! 